\title{
MASS TRANSPORT AND TURBULENCE IN GRAVITATIONALLY UNSTABLE DISK GALAXIES. II. THE EFFECTS OF STAR FORMATION FEEDBACK
}

\author{
Nathan J. Goldbaum ${ }^{1}$, Mark R. Krumholz ${ }^{2,3}$, and John C. Forbes ${ }^{3}$ \\ ${ }^{1}$ National Center for Supercomputing Applications, University of Illinois at Urbana-Champaign, 1205 W. Clark St., Urbana, IL 61801, USA; ngoldbau@illinois.edu \\ ${ }^{2}$ Research School of Astronomy \& Astrophysics, Australian National University, Canberra, ACT 2601, Australia \\ ${ }^{3}$ Department of Astronomy \& Astrophysics, University of California, Santa Cruz, CA 95064, USA \\ Received 2016 January 25; revised 2016 April 29; accepted 2016 May 5; published 2016 August 3
}

\section{ABSTRACT}

Self-gravity and stellar feedback are capable of driving turbulence and transporting mass and angular momentum in disk galaxies, but the balance between them is not well understood. In the previous paper in this series, we showed that gravity alone can drive turbulence in galactic disks, regulate their Toomre $Q$ parameters to $\sim 1$, and transport mass inwards at a rate sufficient to fuel star formation in the centers of present-day galaxies. In this paper we extend our models to include the effects of star formation feedback. We show that feedback suppresses galaxies' star formation rates by a factor of $\sim 5$ and leads to the formation of a multi-phase atomic and molecular interstellar medium. Both the star formation rate and the phase balance produced in our simulations agree well with observations of nearby spirals. After our galaxies reach steady state, we find that the inclusion of feedback actually lowers the gas velocity dispersion slightly compared to the case of pure self-gravity, and also slightly reduces the rate of inward mass transport. Nevertheless, we find that, even with feedback included, our galactic disks selfregulate to $Q \sim 1$, and transport mass inwards at a rate sufficient to supply a substantial fraction of the inner disk star formation. We argue that gravitational instability is therefore likely to be the dominant source of turbulence and transport in galactic disks, and that it is responsible for fueling star formation in the inner parts of galactic disks over cosmological times.

Key words: galaxies: evolution - galaxies: kinematics and dynamics - galaxies: spiral - ISM: kinematics and dynamics - ISM: structure

\section{INTRODUCTION}

The star-forming properties of isolated disk galaxies are driven by two primary effects: gravitational instability and star formation feedback. Both can produce supersonic turbulent motions in an initially laminar disk of gas. This turbulence in turn can lead to non-axisymmetric stresses that mix the interstellar medium (ISM) and transport mass inward and angular momentum outward. We argued in Goldbaum et al. (2015, hereafter Paper I) that such mixing and transport must be an essential component of any explanation for the presentday properties of disk galaxies (also see similar arguments in, for example, Olivier et al. 1991; Ferguson \& Clarke 2001; Krumholz \& Burkert 2010; Forbes et al. 2012, 2014, and Petit et al. 2015). In particular, most present-day Milky Way-sized galaxies lack central holes in their gas distributions (Bigiel \& Blitz 2012), despite the fact that the gas consumption time in their central regions is much shorter than the Hubble time (Bigiel et al. 2008; Leroy et al. 2013), and that most gas accretion either from a hot halo or from the cosmic web is expected to arrive at large galactocentric radii, far from the regions of active star formation (e.g., Dutton 2012; Fraternali et al. 2013). Metallicity gradients in their gas dominated-outer regions are also far too flat to be explained without redistribution of metals from smaller galactocentric radii (Bresolin et al. 2009, 2012; Werk et al. 2011; Yang \& Krumholz 2012; Petit et al. 2015).

Because their effects are similar, it can be difficult to disentangle whether star formation feedback or gravitational instability provides the dominant explanation for any particular aspect of galaxy structure and evolution. In a few cases the attribution is clear. For example, the gas velocity dispersions of galaxies do not decline substantially outside $r_{25}$ (Tamburro et al. 2009; Ianjamasimanana et al. 2012, 2015), despite the almost complete absence of star formation at such large galactocentric radii, a clear sign that star formation feedback cannot be the key physical process there (Agertz et al. 2009, 2015; Bournaud et al. 2010; Krumholz \& Burkert 2010; Forbes et al. 2012, 2014). Conversely, it has long been established by simulations of galaxy disks that gravity alone is incapable of destroying star-forming clouds or producing enough turbulence to yield star formation rates as low as those commonly observed, strongly hinting that star formation feedback is required (e.g., Tasker \& Bryan 2008; Dobbs et al. 2011; Bonnell et al. 2013; Renaud et al. 2013).

In many other cases, however, the effects are not so easy to separate, leading to significant confusion. For example, one class of theoretical models assumes that the velocity dispersion and/or Toomre (1964) $Q$ parameter in galactic disks is regulated by star formation feedback, and use this assumption to deduce a star formation law (e.g., Thompson et al. 2005; Ostriker et al. 2010; Ostriker \& Shetty 2011; Faucher-Giguère et al. 2013). Another class of models assumes that $Q \sim 1$ is maintained by gravitational instability, independent of the star formation law, and instead derive the rate of mass transport through galaxies from this assumption (e.g., Krumholz \& McKee 2005; Dekel et al. 2009; Krumholz \& Burkert 2010; Cacciato et al. 2012; Forbes et al. 2012, 2014; Agertz et al. 2015). Clearly both pictures cannot be entirely correct.

In Paper I, we presented a series of numerical experiments including self-gravity but no stellar feedback, in order to isolate the role of gravitational instability in determining the structure and evolution of disk galaxies. We found that without feedback, star formation rates in our simulations were roughly an order of magnitude too large compared to observed star 
formation rates. However, we found that our model galaxies nonetheless equilibrated to $Q \sim 1$, and to velocity dispersions of $\sim 10 \mathrm{~km} \mathrm{~s}^{-1}$. The energy required to maintain this velocity dispersion and offset the energy loss in radiative shocks came from accretion through the disk of the galaxy. Our fiducial, Milky Way-like model produced a mass inflow rate of $\sim 1 M_{\odot}$ $\mathrm{yr}^{-1}$ throughout the bulk of its disk, sufficient to fuel all the star formation that is observed to occur in the inner disk of a Milky Way-like galaxy. We therefore concluded that gravitational instability alone is capable of fully explaining the observed velocity dispersions and marginal gravitational instability of present-day galactic disks, and that it can explain why star formation does not typically quench in their centers.

In this paper we present a new set of simulations using the same initial conditions presented in Paper I, but now including a novel prescription for star formation feedback. Our goal is to determine how the inclusion of feedback modifies galactic structure and mass transport rates compared to the no-feedback case. Below, we briefly discuss the initial conditions for our simulations (Section 2.1) and describe the theoretical basis and implementation for our feedback model (Section 2.2), which includes all the dominant feedback processes: supernovae (SNe), stellar winds, and photoionization-driven bubbles. This is followed by a discussion of our simulation results, beginning with an overview of the qualitative outcome of our simulations (Section 3.1). Next, we discuss the impact of feedback on the star formation rates and star formation histories (Section 3.2) and ISM structure (Section 3.3) of our model galaxies. Finally, we investigate the evolution of the gravitational instability in our model disks, focusing on the gas velocity structure (Section 3.4.1), Toomre $Q$ parameter (Section 3.4.2), and rate of radial mass transport (Section 3.4.3). We end by reviewing our results and discussing them in context of galaxy formation and models for galactic star formation rates (Section 4).

\section{METHODS}

\subsection{Initial Conditions and Evolution}

To ease comparison with the models run without star formation feedback, in this paper we will be discussing simulations initialized identically to the simulations described in Paper I. This means that the initial portions of the simulations are practically identical to the no feedback cases, up to the formation of the first star particle-any differences are due floating point noise being amplified by dynamical chaos. Briefly, the model galaxies are initialized using the makegalaxy code (Springel et al. 2005). The code makes use of the analytic framework of Mo et al. (1998) to predict the properties of a disk formed in a $\Lambda \mathrm{CDM}$ cosmology given a halo mass, disk mass, and halo spin parameter.

The initial conditions include dark matter and stars, which are modeled as $\mathrm{N}$-body particles, and gas defined on an AMR mesh. The dark matter particles are distributed according to a Hernquist profile, while the stars are distributed in a thin exponential disk and centrally concentrated bulge population. Particle initial conditions are generated by randomly sampling from an analytic distribution function, while the gas is initialized on the AMR mesh again following an analytic azimuthally symmetric exponential density profile.

The parameters of our model galaxies are chosen to loosely match the Milky Way, with a halo mass of $\sim 10^{12} M_{\odot}$, a stellar disk mass of $\sim 10^{10} M_{\odot}$, and a gas mass of $\sim 10^{9} M_{\odot}$. We initialize three different galaxy models with identical parameters besides the initial gas fraction. The low gas fraction (LGF) model begins with a gas fraction of $10 \%$ (relative to the mass of the stellar disk), the fiducial model has a gas fraction of $20 \%$, and the high gas fraction (HGF) model has an initial gas fraction of $40 \%$. We run the LGF and fiducial simulations for 600 Myr. Due to numerical limitations, we have only evolved the HGF run for $300 \mathrm{Myr}$.

The gas, stars, and dark matter are evolved using the Enzo code (The Enzo Collaboration et al. 2014). The gas is evolved using second-order accurate PPM hydrodynamics, and the gravitational potential is evaluated using a multigrid method. Particle dynamics are evoluated using a kick-drift-kick scheme. The maximum spatial resolution of the simulation is $20 \mathrm{pc}$, and the gravitational force resolution is two spatial elements, or $40 \mathrm{pc}$.

\subsection{Star Formation Feedback Model}

Here we describe a novel subgrid model for star formation feedback that includes the effects of ionizing radiation from young stars, winds from evolved massive stars, and the energy and momentum released by individual SN explosions. The parameters for the model are chosen to match a starburst 99 (Leitherer et al. 1999; Vázquez \& Leitherer 2005; Leitherer et al. 2014) model of a young stellar population that fully samples the initial mass function (IMF). Additional details regarding the model can be found in Forbes et al. (2016).

\subsubsection{Stochastic Stellar Population Synthesis}

The first step in our feedback model is the formation of a star particle. The recipe for particle formation is the same as that used in Paper I, so we will not repeat it here. All star particles in our simulations form with a uniform initial mass of $300 M_{\odot}$. Within each of these particles we expect there to be a few stars massive enough to produce SN explosions. We model this based on a scaled down version of a starburst 99 model of a $10^{6} M_{\odot}$ stellar population. For such a massive cluster, we should expect $\sim 10^{4} \mathrm{SNe}$ II over the lifetime of the population. Scaling down to our star particles, we expect to produce $\sim 3$ $\mathrm{SNe}$ per star particle. The true number of $\mathrm{SNe}$ for each particle is chosen randomly at runtime, by drawing from a Poisson distribution with an expectation value set by scaling the $10^{6} M_{\odot}$ starburst 99 model down to our $300 M_{\odot}$ particle mass.

Once we have determined the number of SN explosions the star particle will launch over the course of the simulation, we set the times at which each individual $\mathrm{SN}$ will detonate by drawing from the SN delay time distribution predicted by starburst99. There is a one-to-one mapping between SN delay time and progenitor initial mass, which we make use of to associate a stellar mass to each $\mathrm{SN}$ progenitor. For each $\mathrm{SN}$ progenitor, we also record the expected main sequence ionizing luminosity (Parravano et al. 2003) (implicitly assuming that the ionizing radiation from massive stars that are below the minimum initial mass for a SN II explosion is negligible) which we in turn use for our $\mathrm{H}$ II region feedback model. In practice, all of this data is regenerated at each timestep using unique random number streams seeded by the unique particle ID. This adds extra CPU cost in each timestep while avoiding the memory and communication costs of saving and synchronizing this data for all dynamically created star particles. 
Once the massive stars in each star particle have been identified, we loop over all particles in the simulation, applying various forms of feedback to the hydrodynamic quantities defined on the Enzo AMR mesh.

\subsubsection{H II Region Feedback}

Massive stars emit copious amounts of ionizing radiation, capable of creating bubbles of warm, ionized gas in the densest regions of the ISM. The heating provided by $\mathrm{H}$ II regions can stabilize gas against gravitational collapse, reducing the star formation rate immediately and directly.

We include $\mathrm{H}$ II region feedback in our simulations using the following algorithm. If a grid cell contains a dynamically created star particle containing unexploded massive stars, we increase the gas temperature to emulate photoionization heating. The amount of heating is determined by comparing the volume of the Strömgren sphere, $V_{s}=(4 / 3) \pi R_{s}^{3}$, with the volume of the cell in which the star particle resides, $V_{c}=\Delta x^{3}$, where $\Delta x$ is the local cell spacing. Here,

$$
R_{s}=\left(\frac{3 S}{4 \pi \alpha_{B} n^{3}}\right)^{1 / 3}
$$

is the Strömgren radius, determined by equilibrium between photoionization and recombination at a given density, $S$ is the ionizing luminosity emitted by the star particle, $n$ is the number density of gas in the host cell, and $\alpha_{B}=2.6 \times 10^{-13} \mathrm{~cm}^{3} \mathrm{~s}^{-1}$ is the case- $B$ recombination coefficient, assuming a temperature of $10^{4} \mathrm{~K}$. If $V_{s}>V_{c}$, the cell is heated to a temperature of $10^{4} \mathrm{~K}$. If the cell is already hotter than that, no heating is applied. If $V_{s}<V_{c}$, we apply a volume filling factor correction, heating the cell to $T=10^{4} \mathrm{~K}\left(V_{s} / V_{c}\right)$. If more than one particle contains SN progenitors in any given cell, they each contribute separately, increasing the cell temperature up to a maximum of $10^{4} \mathrm{~K}$.

One major downside of this approach is that it is fully local to any given cell in the simulation. In principle, $\mathrm{H}$ II regions may grow to be larger than a single cell, suppressing $\mathrm{H}$ in region feedback and making it more difficult for heated cells to dynamically affect the state of the simulation. In practice this is not a big concern for the simulations we discuss here, since only the rarest massive star clusters will produce $\mathrm{H}$ II regions with diameters bigger than $20 \mathrm{pc}$. In anticipation of future simulations at higher resolution, we plan to modify this feedback routine to apply $\mathrm{H}$ in heating feedback in a distributed fashion.

\subsubsection{Winds from Massive Stars}

In addition to heating by $\mathrm{H}$ II regions, very massive stars eject a substantial fraction of their envelopes, recycling gas back into the ISM. We include this effect in our feedback algorithm by altering the density and internal energy of gas in cells that contain star particles associated with very young stellar populations. Formally, we use the output of a starburst99 calculation to determine the rate of wind energy and mass injection per unit mass and as a function of age for a stellar population that fully samples the IMF. We then add the appropriate amount of mass and internal energy to each star particle's host cell each time step, until the last SN explosion occurs.
The primary effect of winds is to suppress star formation somewhat, since soon after a star particle forms, the gas in the cell where the particle spawned will heat up. In addition, this captures a significant fraction of the gas recycling from a young stellar population. We do not include the effect of AGB winds, although that is not a significant concern given that AGB stars only become significant after a few Gyr and we evolve our simulations for a maximum of $600 \mathrm{Myr}$. In practice, the star formation suppression effect of winds is minor compared to SN explosions.

\subsubsection{SN Explosions}

$\mathrm{SN}$ explosions are a commonly used source of feedback in simulations of galaxy formation and evolution, including simulations on cosmological scales (Cen \& Ostriker 1992; Springel \& Hernquist 2003; Scannapieco et al. 2006) all the way down to parsec scales (Joung \& Mac Low 2006; Kim et al. 2011a; Kim \& Ostriker 2015). It has been known for many years (Katz 1992) that simply depositing $E_{\mathrm{SN}}=10^{51} \mathrm{erg}$ per $\mathrm{SN}$ as thermal energy in the location of the $\mathrm{SN}$ explosion does not produce strong feedback in low to moderate resolution simulations. Instead, the energy, which must at minimum be shared by all of the gas in a single computational element (typically $10-1000 \mathrm{pc}$ across) is quickly radiated away since the energy from a single $\mathrm{SN}$ is not enough to heat the amount of gas enclosed in such an extended region above $\sim 10^{6} \mathrm{~K}$. Since the cooling time is much shorter at lower temperatures, the gas quickly radiates away the deposited thermal energy before the $\mathrm{SN}$ is able to do $P d V$ work on the surrounding gas. At high $(\sim 1 \mathrm{pc})$ resolution this is less of a concern, but simulating galaxy evolution at such high resolution is extremely expensive.

To circumvent this problem, low resolution simulations typically deposit the SN energy continuously over many timesteps instead of discrete energy-injection events (Smith et al. 2011), possibly also using direct momentum deposition to ensure gas is ejected out of halos (Oppenheimer \& Davé 2008). The combined heating of many SN explosions over millions of years is sufficient to heat the gas in low resolution simulations, at the cost of resolving the fine-grained structure of the SN feedback. In higher resolution simulations such as ours, some have opted to directly resolve the SN blastwaves (Joung \& Mac Low 2006; Joung et al. 2009), temporarily turn off cooling in regions near the blastwave site to avoid over-cooling (Stinson et al. 2006), directly depositing momentum into the simulation (Kim et al. 2011a), or using a hybrid of approaches (Kimm \& Cen 2014; Kimm et al. 2015).

Our approach most closely resembles the hybrid method of Kimm \& Cen (2014) and Kimm et al. (2015). In our algorithm, each SN has an energy budget of $10^{51} \mathrm{erg}$, but this energy is partitioned into two channels, which we refer to as momentum feedback and energy feedback.

The momentum feedback prescription is as follows. First, we identify particles that will produce $\mathrm{SNe}$ in any given timestep. For each SN explosion, we identify the grid cell where the exploding star particle resides and approximate that the explosion happens in the center of the grid cell. This choice substantially simplifies the implementation since we can exploit symmetry to naturally produce a spherically symmetric explosion that conserves momentum by construction. In the future, we plan to use the prescription described in Simpson et al. (2014), which properly handles the problem of creating 
spherically symmetric momentum-conserving blastwaves that are centered at an arbitrary location. In practice our choice to recenter the explosion at the center of the host grid cell leads to a small loss of resolution at scales below our grid spacing. We do not expect a proper treatment of the sub-resolution position of the exploding SN to substantially alter the results discussed below.

Once the cell at the center of the $\mathrm{SN}$ bubble has been identified, we identify the 26 nearest neighbor cells and loop over each neighbor cell, depositing momentum

$$
\Delta p_{\mathrm{SN}}=1.2 \times 10^{4} M_{\odot} \mathrm{km} \mathrm{s}^{-1} \hat{r},
$$

where $\hat{r}$ is the unit vector connecting the center of the $\mathrm{SN}$ host cell to the neighbor cell under consideration. The normalization for $\Delta p_{\mathrm{SN}}$ used here was chosen based on detailed simulations of SN explosions in a turbulent ISM where the net momentum injection into the ISM can be directly measured (c.f. Cioffi et al. 1988; Kim et al. 2011a; Kim \& Ostriker 2015; Martizzi et al. 2015). The total momentum deposited is $3 \times 10^{5} M_{\odot} \mathrm{km} \mathrm{s}^{-1}$, shared equally between each of the 26 neighbor zones. If the SN explodes at the edge of a grid patch, the portion of the explosion that would happen in cells living on a different grid is not included, effectively "cutting off" the SN explosion and abandoning spherical symmetry. This happens relatively rarely, but is a deficiency of our feedback algorithm, albeit one that is commonly used in distributed feedback calculations in the Enzo code (Kim et al. 2011b; Simpson et al. 2014, although in the latter the feedback region is shifted rather than cut off). To avoid producing spuriously fast-moving gas when a SN explodes in the neighborhood of a relatively low-density cell, we limit the maximum change in velocity for the gas in any given cell to $1000 \mathrm{~km} \mathrm{~s}^{-1}$.

Since particles that spawn SN explosions must always live on the maximum refinement level, the mass of cells in the neighborhood of stars that go $\mathrm{SN}$ is independent of the local gas temperature. This means that heating from $\mathrm{H}$ II region feedback and stellar wind feedback does not substantially alter the dynamics of the blastwaves, since they are driven by direct momentum injection. Instead, the primary effect of the stellar wind and $\mathrm{H}$ II region is to provide thermal support in the densest regions of the ISM and produce a more realistic ISM structure (see Section 3.3 for a more detailed discussion of the ISM structure in our simulations).

The energy feedback portion of our algorithm occurs once we have computed the momentum to be added. We record the change in kinetic energy with respect to the simulation's rest frame for each cell that we deposit momentum into. The total net increase in kinetic energy in the cells surrounding the SN host cell are then deducted from the available budget of $10^{51} \mathrm{erg}$ and the balance of the energy is then deposited in the SN host cell as thermal energy. We also adjust the mass and metallicity in the host cell to account for mass injection due to the SN ejecta. In practice, at $20 \mathrm{pc}$ resolution, most of the thermal energy is used to launch the momentum feedback, so the amount available for heating the host cell is much less $10^{51} \mathrm{erg}$. We discuss how this affects our results in Section 3.3, when we note how the hot phase of the ISM is suppressed compared to observed hot phases in nearby star-forming galaxies.

In a small fraction, $\sim 1 \%$, of $\mathrm{SN}$ explosions, the kinetic energy injected by our momentum feedback prescription exceeds $10^{51} \mathrm{erg}$. In these cases, we inject no energy into the SN host cell, but do not go back to redo the momentum injection to ensure detailed energy balance. In practice, this situation happens when a $\mathrm{SN}$ explosion happens in the neighborhood of one or more low-density cells, so we do not expect this effect to impact our conclusions regarding the destruction of dense star-forming clouds.

Before moving on, we pause to note that one advantage of our feedback prescription is that, at least in its treatment of the cold ISM, it should not be tremendously sensitive to resolution. This is because the effects of the gas on the cold ISM come mainly from the momentum feedback, and the total amount of momentum we are injecting is resolution-independent. Since momentum cannot be radiated away, and nothing behaves nonlinearly with density (as does, for example, the rate of radiative cooling), the volume into which the momentum is injected does not affect the results as strongly as it would for an energydriven feedback prescription. This statement does not apply to the energy feedback portion of our prescription, however, a point that will become relevant in Section 4.2.

\section{RESULTS}

Here we describe the results of our simulations. First, in Section 3.1, we focus on the qualitative evolution of our simulated disks, focusing on their morphology. Next, in Section 3.2 we describe the impact of our feedback algorithm on the star formation rates and star formation histories. In Section 3.3 we describe the structure of the ISM in our simulated galaxies, showing how feedback moderates the amount of gas available for star formation by dispersing dense gas concentrations. Finally, in Section 3.4 we discuss the development of gravitational instability via measurements of the turbulent velocity structure, Toomre Q parameter, and the mass transport rate.

Throughout this section, all the derived quantities we measure are computed exactly as in Paper I, and we refer readers to the Appendix of that paper for a full description of our analysis pipeline. The source code for the pipeline and supplementary analysis scripts are available on Bitbucket. ${ }^{4}$ The raw simulation data, initial conditions, and ancillary postprocessed data are also available for download. ${ }^{5}$

\subsection{Qualitative Outcome}

We present snapshots of the fiducial simulation at four times in Figure 1. The snapshot displays the gas and stellar surface density and effective sound speed, as well as $Q_{\text {total }}$, which includes contributions from both gas and stars. The outcome of all three simulations are similar to the evolution depicted in Figure 1, so we will describe the features shared between the three simulations here and explain how the results vary as a function of gas fraction below.

Early in the simulation, the morphology is dominated by circular, expanding rings of gas and stars, which we interpret as a manifestation of unphysical transient behavior as the disk settles out of its simplified initial conditions. Later, the rings dissipate, giving way to prominent spiral arms. The arms form spontaneously, and are clearly visible in both the gas and stellar surface density maps.

\footnotetext{
4 https://bitbucket.org/ngoldbaum/galaxy_analysis

5 http://dx.doi.org/10.13012/J85Q4T1T
} 


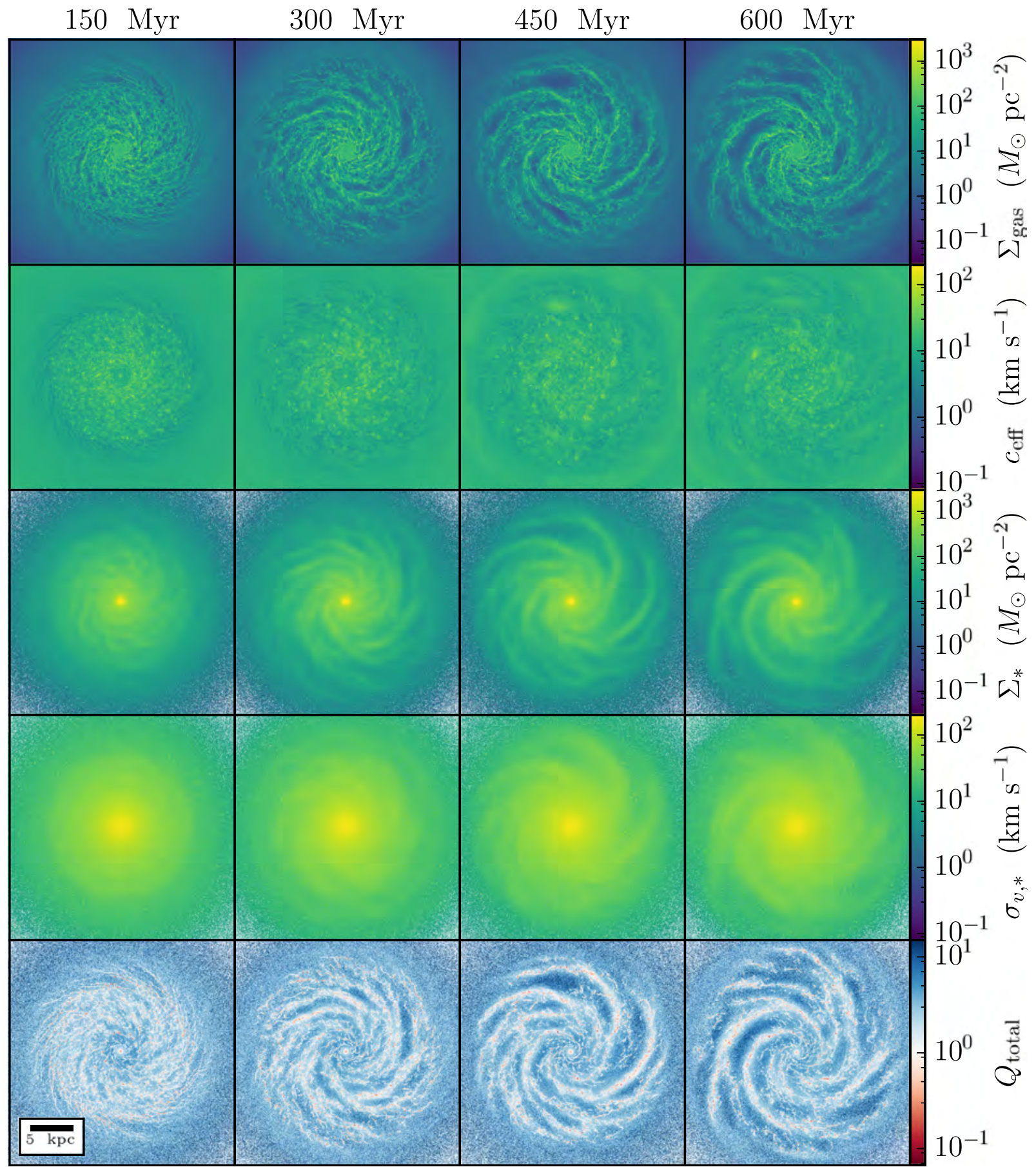

Figure 1. Surface density and vertically averaged effective sound speed for both the gas and stars, as well as the combined Toomre $Q$ parameter after $150,300,450$, and $600 \mathrm{Myr}$ has elapsed in the fiducial simulation.

The gas in the inner disk is concentrated in thin filaments that are continuously sheared, leading to a tightly wound spiral pattern. At intermediate radii, the filaments break up into individual isolated clouds. This morphology develops as a smooth transition along the prominent spiral arms at the edge of the star-forming disk. Gas in the outer disk is smoother, only collapsing into more or less isolated clouds toward the end of the simulation. Individual SN explosions may also temporarily evacuate the area around the explosion site. This is visible in the gas density, in the upper right quadrant of the disk in the snapshot at $T=300 \mathrm{Myr}$ in
Figure 1, where several blastwaves have evacuated regions embedded in a spiral arm.

The gas effective sound speed is roughly constant with radius, in an azimuthally averaged sense, although with significant variation at any given radius. Individual SN explosions produce a substantial amount of hot gas in the inner disk, with $c_{\text {eff }}$ as high as $\sim 50-100 \mathrm{~km} \mathrm{~s}^{-1}$ in interarm regions. Dense gas collects in filaments, where $c_{\text {eff }}$ is suppressed compared to the interarm regions, with $c_{\text {eff }} \sim$ $1-10 \mathrm{~km} \mathrm{~s}^{-1}$. We will show in Section 3.4.1 that the effective sound speed in the dense gas is dominated by the turbulent 
velocity dispersion. In the outskirts of the galaxy, SN-heated regions become rarer. Here the interarm gas is still heated to $10-20 \mathrm{~km} \mathrm{~s}^{-1}$, and appears similar to the interarm gas seen in the simulations without feedback.

The stellar component is substantially smoother, albeit morphologically similar to the gas on kpc scales. Once the disk has settled, the stars show a prominent $m=5$ spiral pattern. The stellar velocity dispersion does not vary much over the course of the simulation, showing neither appreciable heating nor dissipation. For this reason, the distribution of $Q_{\text {total }}$ is primarily dictated by the behavior of the gas. Early in the simulation, as the disk comes into equilibrium, $Q_{\text {total }} \simeq 1-2$, with $Q_{\text {total }} \lesssim 1$ in the highest gas density regions and $Q_{\text {total }} \simeq 1$ in the interarm regions. Later in the simulation, $Q_{\text {total }}$ increases in the interarm regions, reaching as high as $\sim 5$ in inter-arm regions, while remaining $\sim 1$ within the spiral arms.

We can see the remarkable effect of feedback on the structure of our simulated galaxies by comparing directly with the runs with no feedback, as shown in Figure 2. At late times in the runs with no feedback the morphology is dominated by long-lived bound clumps, while in the runs with feedback the gas is distributed relatively smoothly, although it is modulated by the spiral pattern. Since gravitationally bound clouds are efficiently destroyed by SN feedback, the stellar distribution is also substantially more smooth, showing no clear concentrations of stars besides the bulge population present in the initial conditions. The gravitational stability parameter $Q_{\text {total }}$ is also more smoothly varying in the runs with feedback

When comparing the LGF and fiducial simulations with feedback, we see the disks look very similar. The star-forming region is smaller in the LGF case since the outskirts of the disk have not had time to collapse, but the inner disks are practically indistinguishable. We do see some differences in the effective sound speed, which is somewhat higher in the inner portion of the fiducial simulation. This difference between the fiducial and LGF simulation is driven by the substantially higher star formation rate in the fiducial simulation producing more gas heated by both photoionization and SN thermal feedback.

None of our simulations produce significant galactic winds. This may be due to the resolution of our models, but might also be due to the masses of our simulated disks. We discuss this point in more detail in Section 4.2.

\subsection{Star Formation}

Star formation feedback has a profound effect on the star formation rates in our model galaxies. In Figure 3, we show the star formation history of our galaxy simulations as measured by binning the dynamically created star particles present at the end of each simulation by creation time. We show models run with and without feedback for all three choices of initial gas fraction.

Initially, the models are identical: the star formation rate shows a peak followed by a decline. This pattern is driven by the dynamical nature of the initial collapse of the disk, which is identical in the cases with and without feedback up to the formation of the first star particle. However, in both the fiducial and HGF cases, the initial peak is somewhat depressed and the following trough in the star formation history is deeper. In the LGF case the star formation histories agree for a somewhat longer period, diverging only as the star formation rate begins to increase again.

Eventually, for the LGF and fiducial cases, the star formation rates in the simulations with feedback converge to a quasi- equilibrium value. The HGF case also appears to be converging to an equilibrium value, although it has not run long enough to fully converge. For the LGF, fiducial, and HGF cases, the star formation rates converge to $\sim 0.3 M_{\odot} \mathrm{yr}^{-1}, \sim 2 M_{\odot} \mathrm{yr}^{-1}$, and $\sim 10-20 M_{\odot} \mathrm{yr}^{-1}$, respectively. For the runs with feedback, we do not see the long-term decrease in the star formation rate seen in the runs with no feedback. Those decreases are primarily driven by gas depletion, and since the star formation rate is substantially depressed in the simulations with feedback, there is not sufficient time over the course of our simulations to substantially deplete the gas.

The equilibrium star formation rate scales super-linearly in the gas fraction. The superlinearity has two causes that we can identify. First, as we discuss further in Section 3.3, the runs with higher gas fractions have higher fractions of their ISM in cooler phases that lack thermal support and are liable to undergo star formation. This is primarily a density effect: runs with higher gas fractions have higher midplane densities, and this shifts the balance between radiative cooling $\left(\propto n^{2}\right)$ and heating $(\propto n)$ more in favor of cooling. Second, a higher gas fraction also raises the midplane pressure, and this in turn raises the density of the cold, star-forming gas. The increase in density in turn lowers the dynamical time in this gas, allowing it to form stars faster.

In comparing to observations, it is also helpful to consider the star formation rate point by point, rather than summed over the entire galaxy. To characterize the star formation behavior on such smaller scales, it is helpful to consider the depletion time

$$
t_{\text {dep }}=\frac{\Sigma_{\text {gas }}}{\Sigma_{\text {sfr }}},
$$

the time it would take to consume all of the gas at a given position in a galaxy. Resolved observations of nearby starforming galaxies indicate typical depletion times in the molecular phase of $\sim 2 \mathrm{Gyr}$, with approximately a third of a decade of scatter (Bigiel et al. 2008, 2011; Leroy et al. 2013).

We show the azimuthally averaged depletion time as a function of radius and time in our simulations in Figure 4. We see that, in comparison to observations, our model galaxies have somewhat lower depletion times, with $t_{\mathrm{dep}}=0.5-1.5 \mathrm{Gyr}$ throughout most of the star-forming disk over the full course of all three simulations. In the inner galaxy, $t_{\text {dep }}$ is lower, $\sim 200$ Myr. There is some observational evidence for such a decline in depletion time in the very centers of galaxies (Leroy et al. 2013), but by less than occurs in our simulations. We also note that the time evolution of the azimuthally averaged depletion time shows relatively little structure, particularly in the LGF case. There is substantially more variation at a fixed time in our simulations, but performing an azimuthal average washes out most of the extreme variations due to the formation of star-forming clouds and evacuated inter-arm regions. In the fiducial case we also see that the depletion time is suppressed somewhat in spiral arms, particularly at early times. There is some observational evidence that molecular gas in regions of high shear have longer depletion times (Meidt et al. 2013), and our simulations appear qualitatively consistent with this finding. However, we note that mechanism proposed by Meidt et al. (2013) for changing the depletion time-variations in the cloud surface pressure between low- and high-shear regions leading to variations in the force balance within molecular clouds-appears unlikely to explain the results in our 
No Feedback

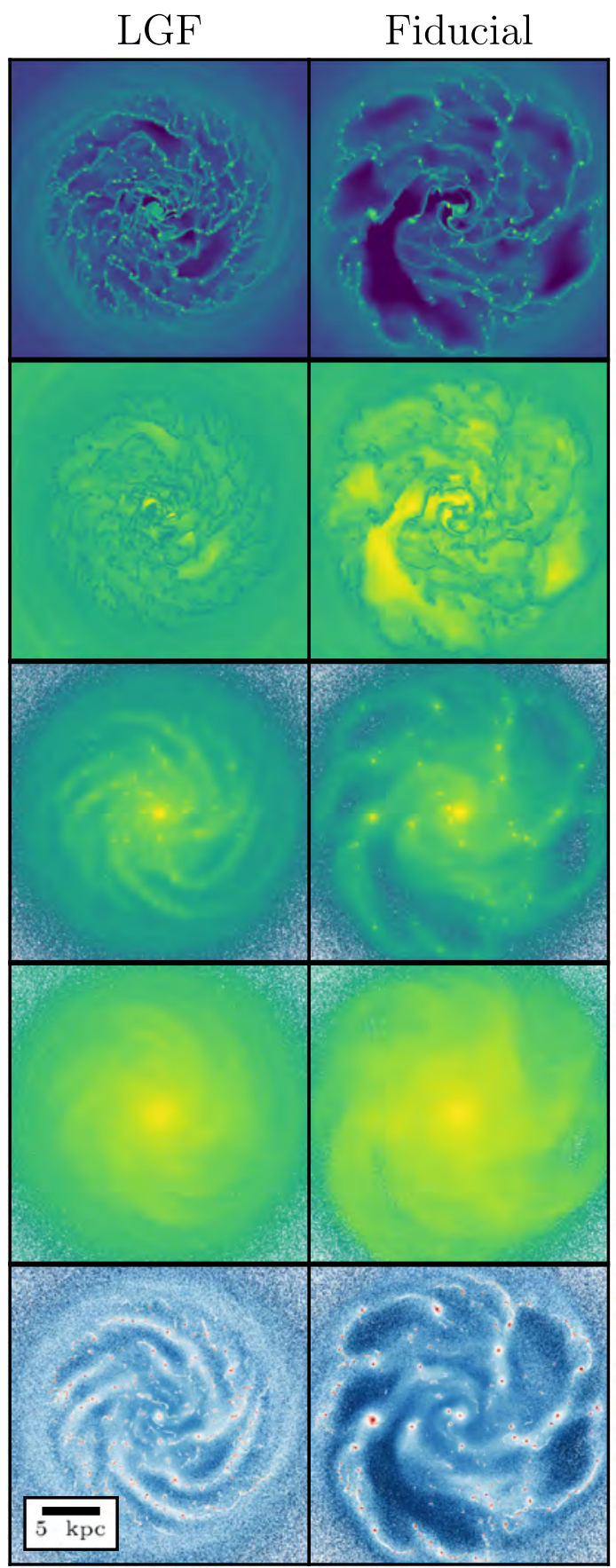

\section{Feedback}

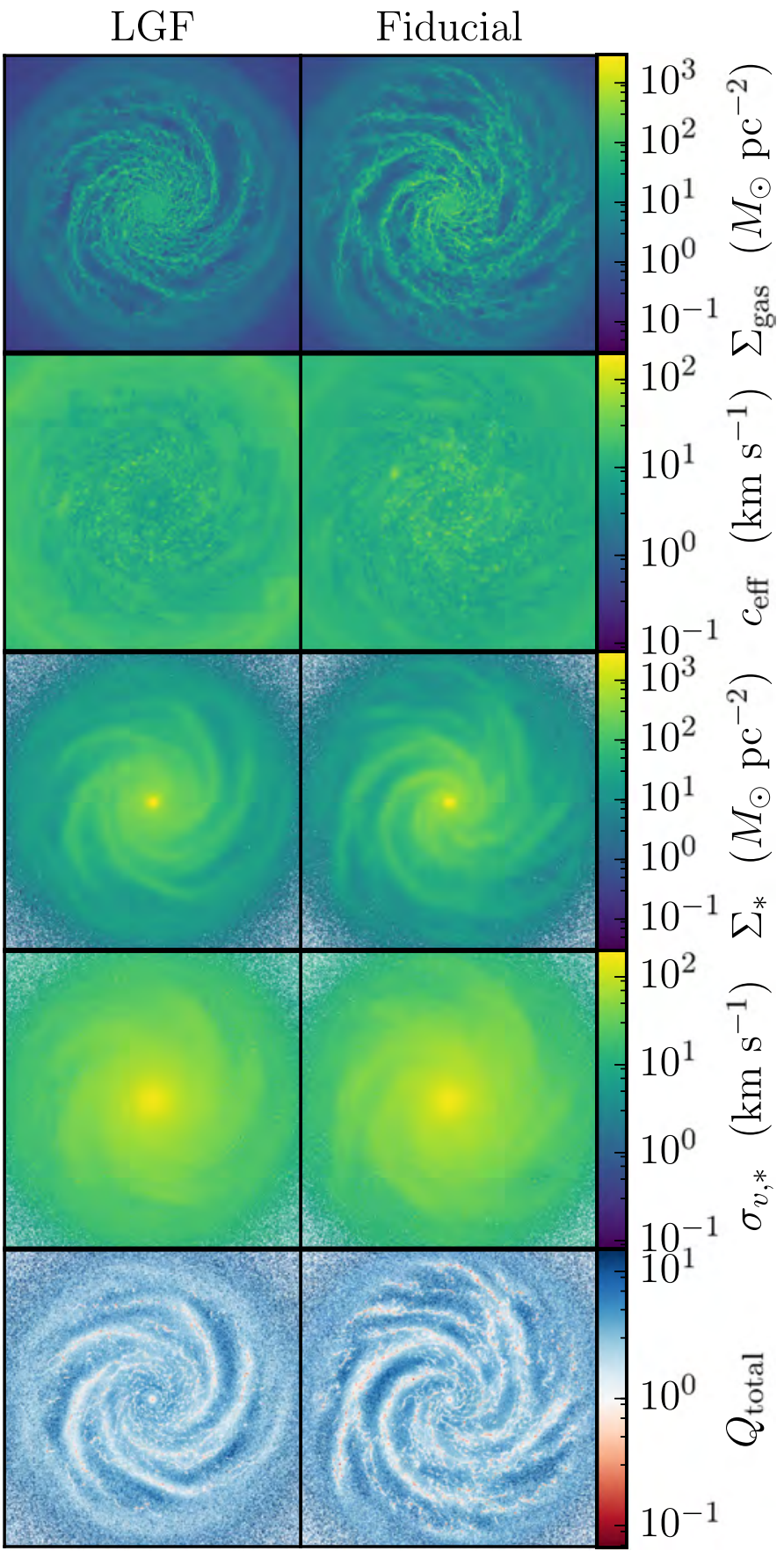

Figure 2. Same as Figure 1, but showing a fixed simulation time $(T=600 \mathrm{Myr})$ for four simulations. We show both the low gas fraction (first and third columns) and fiducial (second and fourth column) cases with (left two columns) and without feedback (right two columns). The high gas fraction simulations are not included since the run including star formation feedback was only evolved for 300 Myr. The simulations with no feedback are described in Paper I.

simulations, since our resolution is insufficient to capture the internal structure of molecular clouds.

To guide our intuition, we can also directly compare to observations of resolved star formation in nearby galaxies. This is shown in Figure 5, which can be directly compared to Figure 4 of Bigiel et al. (2008), Figure 1 of Leroy et al. (2013), or Figures 2 and 3 of Krumholz (2014). To generate this plot, we first generate maps of the $\mathrm{H}_{2}$ and $\mathrm{H}_{\text {I }}$ surface densities by making use of the analytic approximations of Krumholz et al. (2008, 2009), and McKee \& Krumholz (2010). These approximations are based on detailed calculations of selfshielding in idealized cloud complexes and can predict the molecular gas fraction $f_{\mathrm{H}_{2}}=\Sigma_{\mathrm{H}_{2}} / \Sigma_{\text {gas }}$ as a function of $\Sigma_{\text {gas }}$ and metallicity. In practice, we use the formula given in Equation (93) of McKee \& Krumholz (2010),

$$
f_{\mathrm{H}_{2}}=1-\frac{0.75 s}{1+0.25 s}
$$




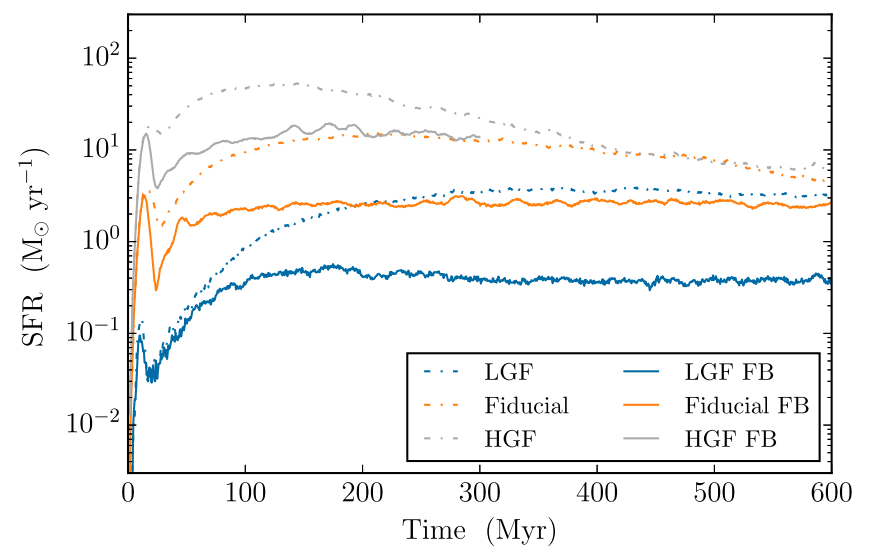

Figure 3. Star formation rate history for our simulated galaxy models. We show the low gas fraction (blue), fiducial (orange), and high gas fraction (gray) cases, both with (solid lines) and without (dashed lines) star formation feedback.

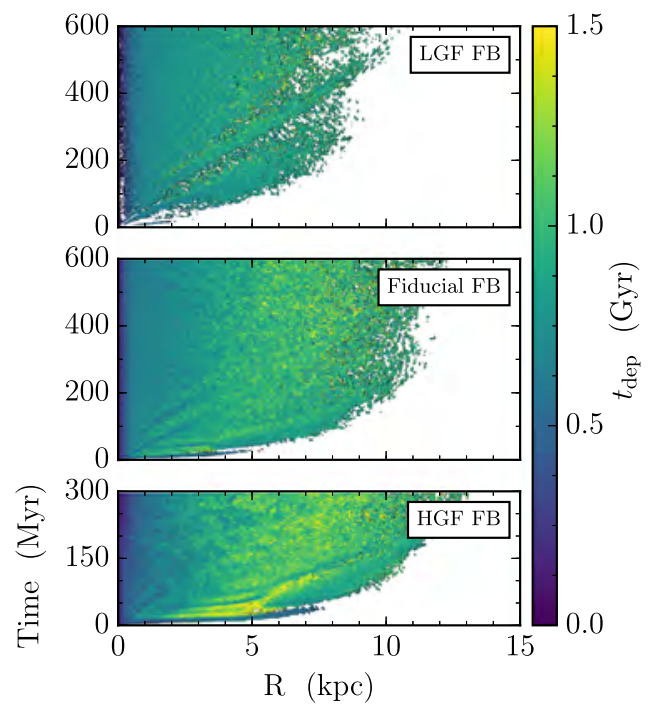

Figure 4. Azimuthally averaged gas depletion time $\left(t_{\mathrm{dep}}=\Sigma_{\mathrm{gas}} / \Sigma_{\mathrm{SFR}}\right)$ as a function of radius and time.

where

$$
s=\frac{\ln \left(1+0.6 \chi+0.01 \chi^{2}\right)}{0.6 \tau},
$$

$\chi=0.77\left(1+3.1 Z^{0.365}\right), \tau=0.066 Z \Sigma_{\mathrm{gas}} /\left(M_{\odot} \mathrm{pc}^{-2}\right)$, and $Z$ is the metallicity relative to the solar value. Our simulations are initialized at solar metallicity and do not show significant metallicity evolution so we assume $Z=1$ when evaluating Equation (5). We generate maps of $\Sigma_{\mathrm{SFR}}$ by projecting the expected star formation rate along the $z$-axis, given our star formation law.

Since $f_{\mathrm{H}_{2}}$ is a function only of the gas surface density, we can immediately calculate

$$
\Sigma_{\mathrm{H}_{2}}=f_{\mathrm{H}_{2}} \Sigma_{\mathrm{gas}}
$$

and,

$$
\Sigma_{\mathrm{HI}}=\Sigma_{\text {gas }}-\Sigma_{\mathrm{H}_{2}} \text {. }
$$

The latter expression ignores the contributions of ionized gas, which we will show is a good approximation in Section 3.3, and assumes the mass fraction of metals is negligible.
To directly compare with observations, which are typically done with $\sim \mathrm{kpc}$ scale resolution elements (although see Schruba et al. 2010 and Onodera et al. 2010), we degrade the resolution of our surface density maps to $740 \mathrm{pc}$, matching the resolution of the THINGS survey (Bigiel et al. 2008; Leroy et al. 2008). Next, we take each pixel at each simulation time in our degraded resolution surface density maps to be an independent measurement of the surface density of atomic hydrogen, molecular hydrogen, and star formation. We combine the set of all measurements we infer from all of our simulation snapshots, and create a 2D histogram, which we plot in Figure 5.

In all three cases, the classical Kennicutt-Schmidt law (Kennicutt 1998; Kennicutt \& Evans 2012), where the total gas surface density is on the $x$-axis, shows a super-linear scaling, as observed in nearby star-forming galaxies. The molecular gas Kennicutt-Schmidt law is shallower, showing a roughly linear scaling at the low surface density end, moving to a superlinear scaling at the high surface density end. Lastly, as observed in nearby galaxies (Bigiel et al. 2008), the $\mathrm{H}$ I surface density shows no correlation with the surface density of star formation.

In both the fiducial and HGF simulations, we see a transition to a low depletion time mode of star formation at the high gas surface density side of the phase space. This may correspond to a "starburst" mode of star formation (cf. Daddi et al. 2010), or may simply indicate that our feedback prescription is inefficient at destroying high surface density clouds, allowing star formation to proceed there for longer than in lower surface density conditions.

\subsection{ISM Structure}

As we showed in Section 3.1, particularly in Figure 2, our feedback model has a dramatic effect on the equilibrium structure of the ISM in our model galaxies. Rather than ending up in a state where the bulk of the gas collects into massive gravitationally bound clouds, the gas is instead more smoothly distributed throughout the disk, only collecting in large-scale spiral arm patterns.

We can make this statement quantitative by segmenting the gas in our simulated galaxies according to ISM phase. In Figure 6, we show an example temperature-density phase diagram, with various ISM phases marked as cross-hatched regions. The regions we identify can primarily be separated into two components: equilibrium and non-equilibrium phases. The former, which include the warm neutral medium (WNM), thermally unstable phase (Unstable), cold neutral medium (CNM), and star-forming gas (SF), all fall along the equilibrium cooling curve, i.e., the region of phase space where, at a fixed density, cooling (primarily due to metal line emission) and heating (primarily due to thermalization of photoelectrons ejected off of dust grains) exactly balance (Field et al. 1969; Wolfire et al. 2003). In practice, these equilibrium regions have a finite width due to small-scale density and temperature fluctuations, so we also include a narrow vertical range above and below the equilibrium curve for each equilibrium component. Non-equilibrium phases, including gas heated by $\mathrm{H}$ II region feedback and SN thermal feedback, fall above the equilibrium cooling curve. Note that while we do not explicitly include a prescription for the formation of molecular hydrogen, our star formation threshold corresponds approximately to the transition to the molecular phase, and so 

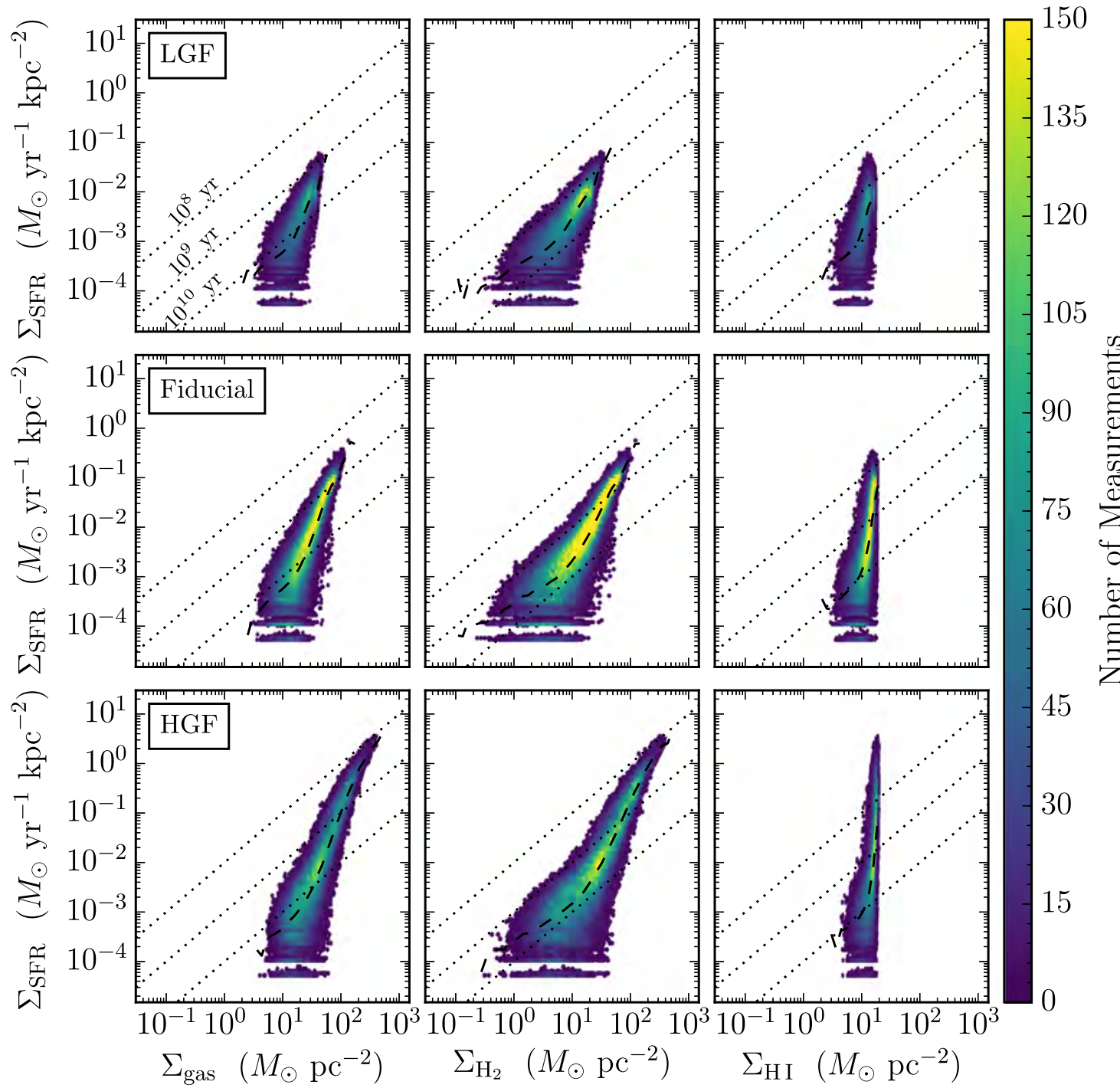

Figure 5. Star formation rate surface density as a function of gas (left column) $\mathrm{H}_{2}$ (middle column) and $\mathrm{H}$ i (right column) surface density. We show data from the low gas fraction (top row), fiducial (middle row) and, high gas fraction (bottom row) models. To guide the eye, we also mark lines of constant depletion time ( $10^{8}, 10^{9}$, and $10^{10}$ years) as dotted lines.

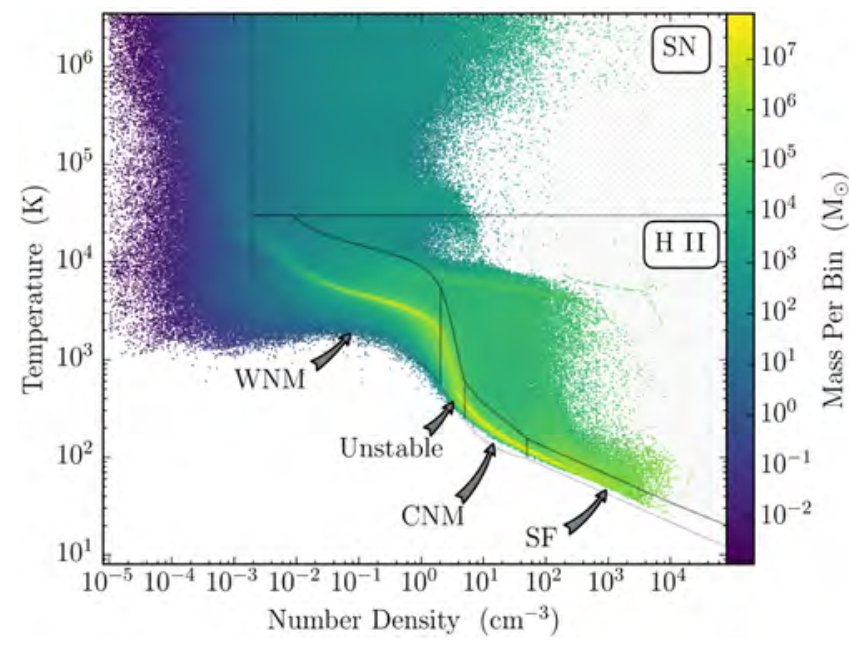

Figure 6. Density-temperature phase diagram for the gas in the fiducial simulation at $T=600 \mathrm{Myr}$. We indicate the regions of phase space we use to define various components of the ISM. our star-forming phase can be thought of as corresponding to the molecular gas phase in a real galaxy.

We can quantitatively compare the impact of star formation feedback on the structure of the ISM by finding the gas mass in each ISM component as a function of time for each of our simulated galaxies. The results of this comparison are shown in Figure 7. We show simulations without (left column) and with (right column) feedback, for each choice of initial gas fraction.

In all three simulations without feedback, the bulk of the gas mass is locked up in dense star-forming gas. Over the course of the simulation, this gas is converted into star particles, until eventually the gas supply is exhausted. While there is still substantial gas left at the end of the simulation in the LGF run, the bulk of the gas in fiducial and HGF is converted into stars over the course of the simulation.

The story is markedly different in the simulations with feedback. Rather than being locked up in star-forming gas, the bulk of the ISM is in the WNM or CNM. In the LGF case, the gas is approximately evenly split between WNM and CNM, with a smaller fraction ending up as star-forming gas. In the 


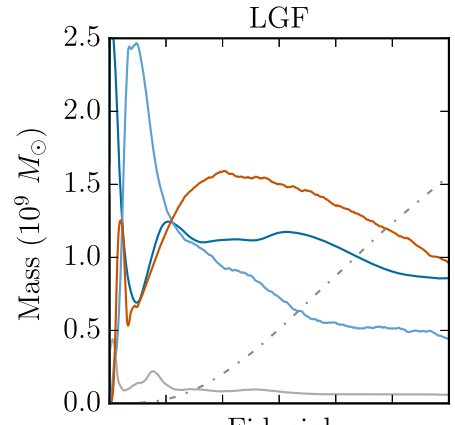

Fiducial

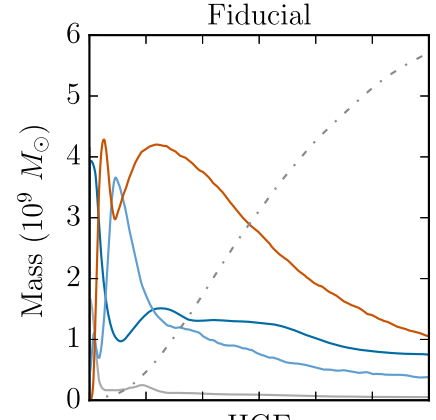

HGF
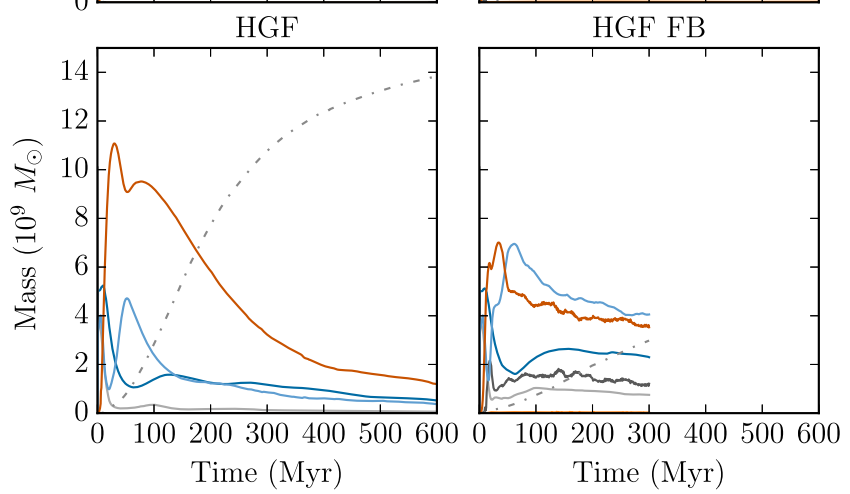

Figure 7. Time evolution of the masses of the ISM components depicted in Figure 6 (solid lines) along with the mass of dynamically formed stars (dotteddashed line). The left column shows simulations without feedback, while the right column shows those with feedback.

fiducial and HGF cases, the bulk of the gas ends up in the CNM and star-forming gas is a substantially larger fraction of the ISM mass compared to the LGF run. In both the fiducial and LGF cases, the ISM develops an equilibrium configuration, where the mass of each component is approximately constant over timescales of several hundred Myr. While the mass of gas heated in the $\mathrm{H}$ II heated phase is non-negligible in the runs with feedback, this gas is small fraction of the overall mass of the ISM. In all cases the mass of SN heated gas is negligible.

\subsection{Gravitational Instability}

Here we focus on the gravitational instability that develops in our simulated galaxies. In Section 3.4.1, we focus on the velocity structure in the gas. This is followed in Section 3.4.2 by a discussion of the evolution of the Toomre $Q$ parameter in our simulated disks, showing how an equilibrium value of $Q_{\text {total }}$ naturally develops. Lastly, in Section 3.4.3, we measure the mean radial mass transport rate, and compare it with the star formation rate.

\subsubsection{Velocity Structure}

As we showed above, when we include feedback the gas in our simulated galaxies undergoes a cycle of collapse into
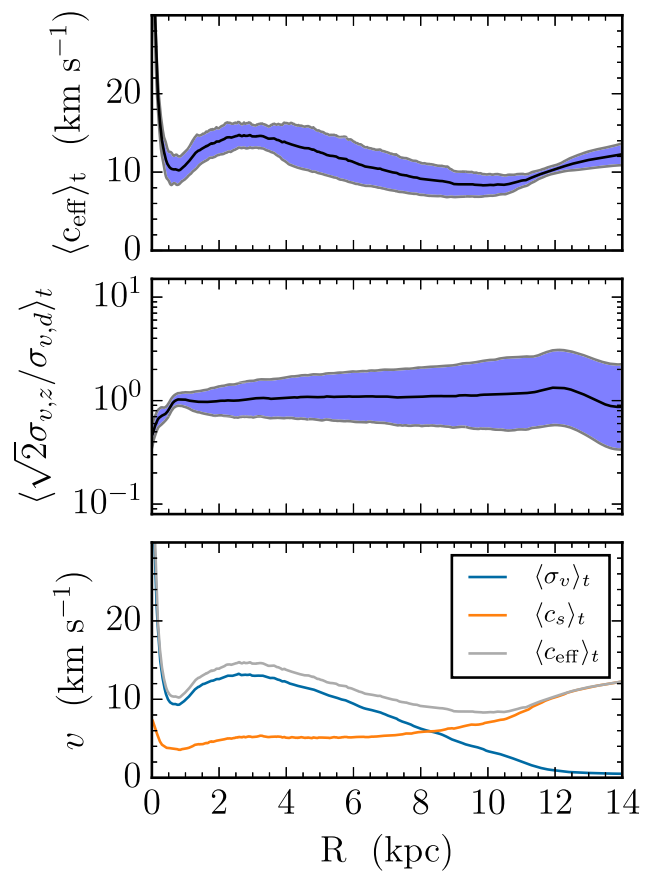

Figure 8. Time-averaged gas effective sound speed (top panel), time-averaged velocity dispersion anisotropy (middle panel), and the contribution to the effective sound speed by the velocity dispersion and thermal sound speed (bottom panel) for the fiducial simulation. The blue shaded regions in the top two panels indicate $1 \sigma$ variance of the plotted quantity as a function radius.

gravitationally bound clouds, rarefaction due to SN feedback, followed by re-collapse into gravitationally bound clouds. Both SN explosions and local departures from a purely axisymmetric gravitational potential generate substantial turbulent velocity dispersions. In addition, SN explosions, winds from massive stars, and $\mathrm{H}$ II regions can heat the gas, providing support for the gaseous disk in the form of thermal pressure.

We can see the typical velocity structure in the gaseous disk by inspecting Figure 8 , where we plot the time average of the gas effective sound speed, sound speed, turbulent velocity dispersion, and the anisotropy in the turbulent velocity dispersion as a function of galactocentric radius. At all radii, $c_{\text {eff }} \gtrsim 8 \mathrm{~km} \mathrm{~s}^{-1}$, reaching as high as $15 \mathrm{~km} \mathrm{~s}^{-1}$ at $R=3 \mathrm{kpc}$. In the inner, star-forming portion of the disk, the effective sound speed is mostly due to turbulent motions, while the sound speed dominates in the outer disk. The mass-weighted sound speed is lower in the inner disk because a typical parcel of gas in the star-forming inner disk will have a higher density, and thus be able to cool more effectively than gas in the outer disk. Compared to the simulations without feedback (see e.g., Figure 9 of Paper I), the effective sound speed is somewhat lower, perhaps surprisingly as the turbulent velocity dispersions of disk galaxies are often thought to be due to feedback. We discuss this point further in Section 4.1.

On the other hand, a major effect of feedback is to make the turbulent velocity field isotropic across the bulk of our simulated galaxies, with $\sqrt{2} \sigma_{v, z} / \sigma_{v, d} \simeq 1$ at all radii. We can also see this as a function of time in Figure 9.

Compared to the runs with no feedback, there is more power in the out-of-disk component of the turbulent velocity dispersions, producing substantially larger gas scale heights in these simulations. Rather than all of the gas collapsing into an extremely thin disk, gas extends both above and below the 


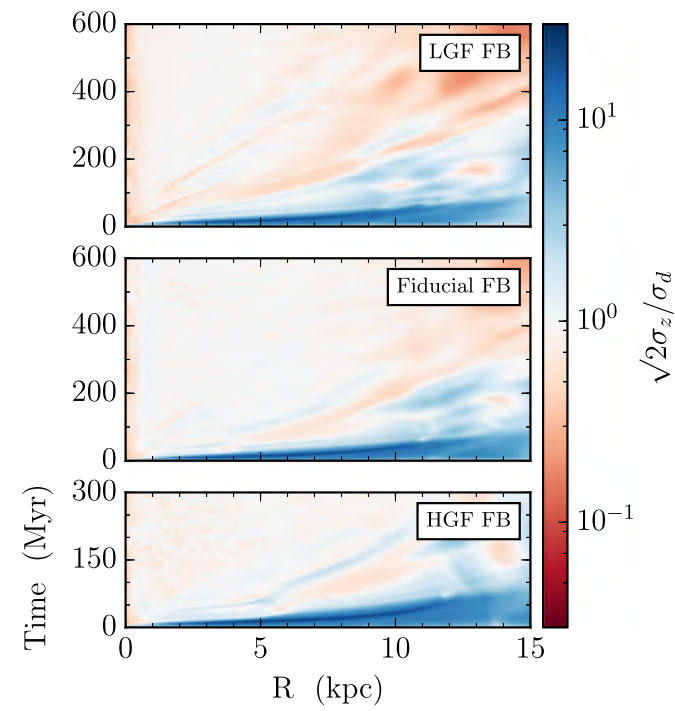

Figure 9. Gas velocity dispersion anisotropy as a function of radius and time.

disk as it gets thrown out of the midplane by feedback. Typically, we find scale heights of several hundred parsecs.

\subsubsection{Toomre $Q$}

The susceptibility of a disk to gravitational instability can be characterized via the Toomre $Q$ parameter. We are able to calculate a combined $Q_{\text {total }}$ that includes the separate contribution to the stability of the disk from both gas and stars, and accounts for the finite thickness of the gaseous and stellar disks (Romeo \& Wiegert 2011). We found in Paper I that, in the absence of feedback, the galaxy initially stabilizes at $Q_{\text {total }} \approx 1$, but that as the simulation proceeds and gas is exhausted, $Q_{\text {total }}$ gradually rises.

We see from Figure 10 that in our runs including stellar feedback, $Q_{\text {total }}$ is remarkably stable, showing little variation with radius or time. There is some modulation at the factor-oftwo level due to surface density fluctuations-transient rings early in the simulation and spiral density waves later on-but we do not see order of magnitude variations as in the runs without feedback.

Stellar feedback is able to slow down the runaway gravitational instability that would otherwise take hold by efficiently destroying gravitationally bound clouds. The disk attains a quasi-stable state, with $Q_{\text {total }} \gtrsim 1$ throughout the disk. Both the gaseous and stellar components show little variation over the course of the simulation, as we see in Figure 11. The gaseous and stellar surface density profile remain smooth, varying exponentially with radius at all times.

We see that stellar feedback is necessary to prevent the runaway fragmentation of star-forming gaseous disks, although the story is somewhat different from the naive expectation that feedback drives turbulence. Instead, feedback moderates the consumption of gas, keeping $Q_{\text {total }}$ close to unity, rather than being driven to a high value by the exhaustion of gas. Crucially, and contrary to assumptions commonly made in theoretical models, this means that feedback actually lowers rather than increases $Q_{\text {total }}$ on galaxy-average scales. Only on the scales of individual molecular clouds, where simulations without feeback reach $Q_{\text {total }} \lesssim 1$, does feedback increase $Q_{\text {total }}$, by dispersing dense clouds via SN blastwaves.

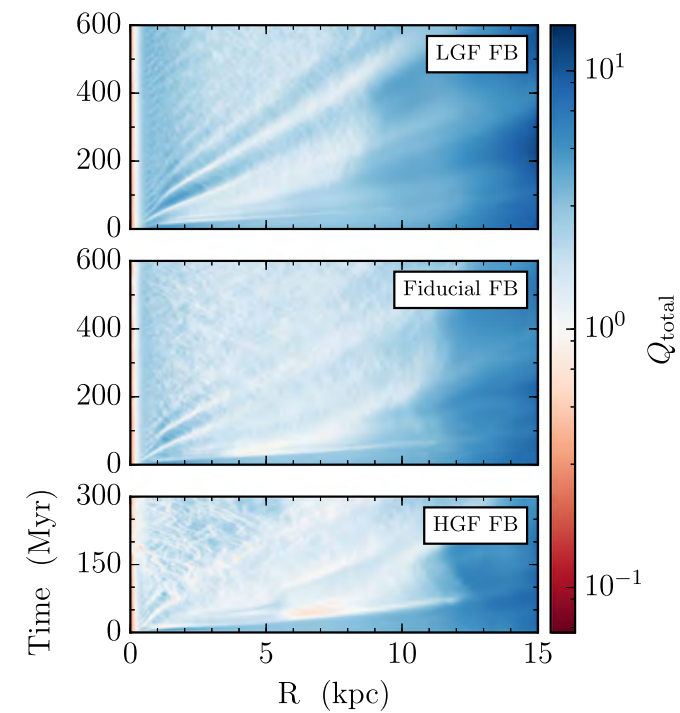

Figure 10. Combined Toomre $Q$ parameter as a function of radius and time.

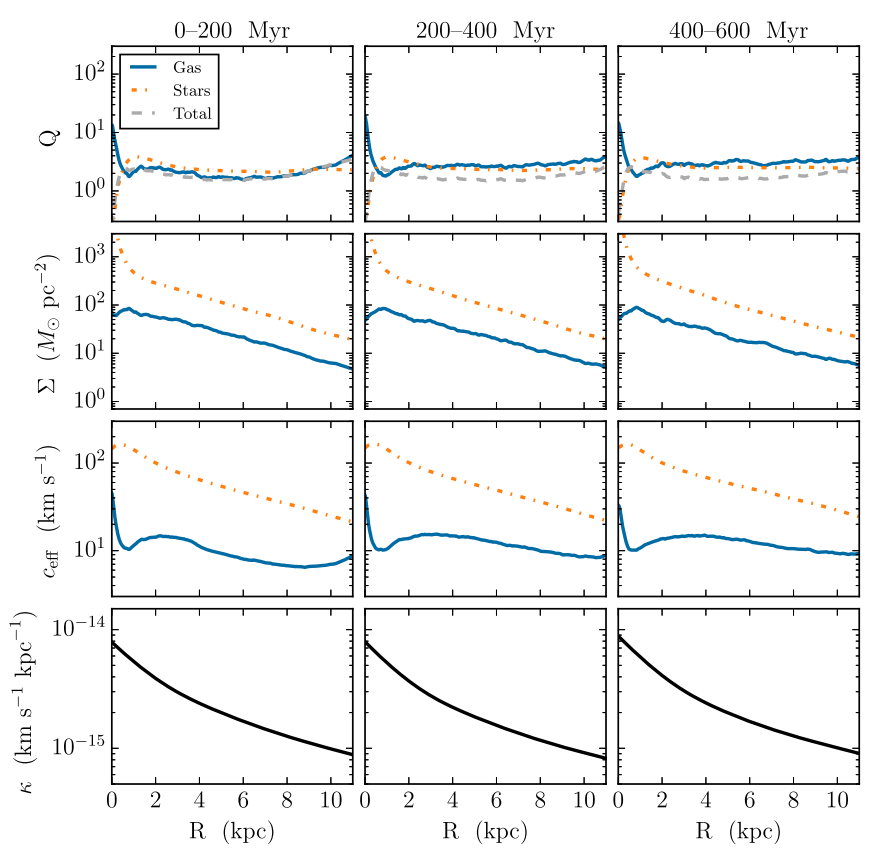

Figure 11. Gas, stellar, and total Toomre $Q$ parameter (top row), gas and stellar surface density (second row), gas and stellar effective sound speed (third row) and epicyclic frequency (bottom row) for the low gas fraction simulation.

\subsubsection{Mass Transport}

Finally, we focus on the radial mass transport rate in the gaseous disk. In the simulations without feedback, we found that mass transport was dominated by $N$-body migration of individual giant clumps. We also found that there was a net radial inflow of gas. While the inflow rate was sufficient to power the observed star formation rates of Milky Way-like galaxies, it was insufficient to supply the rapid star formation in the simulations without feedback.

The runs with star formation feedback show a very different history. In Figure 12, we show the time evolution of the radial mass flux. Compared to the runs without feedback, the early evolution is similar. At early times, the gas mass flux is dominated by rings of inward and outward flux. Since we are 


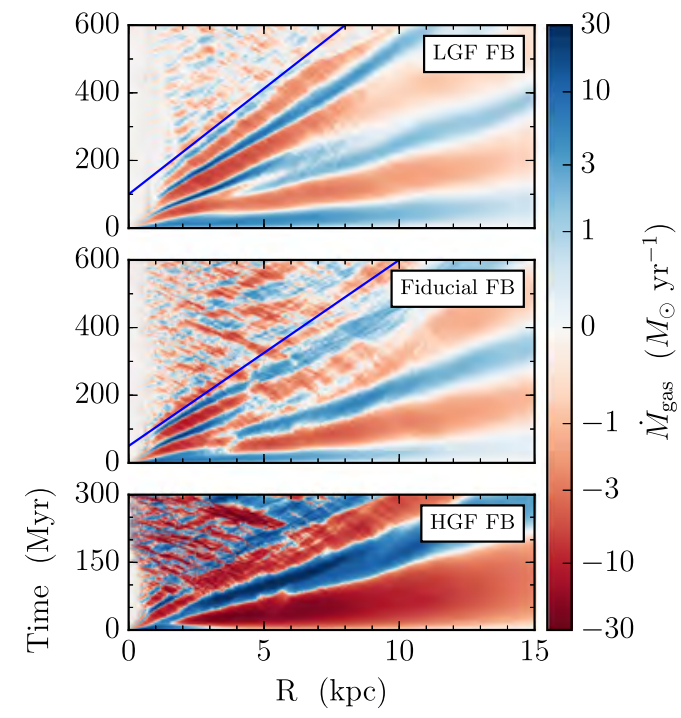

Figure 12. Radial mass flux as a function of radius and time. Negative mass fluxes correspond to radial inward flow, while positive mass fluxes correspond to radial outward flow. The blue lines indicate the wedge averaging region used to produce Figures 8 and 13.

mostly interested in the behavior of the disks after they have settled down into statistical equilibrium, we do not consider the portions of radius-time phase space that are strongly influenced by initial transients (indicated by the blue lines in Figure 12). In the inner disk $(R \lesssim 2 \mathrm{kpc}$ for the LGF case and $R \lesssim 1 \mathrm{kpc}$ for the fiducial simulation), there is very little mass transport. This is not surprising: our galaxies have realistic stellar bulges, and as a result the shape of the rotation curve strongly suppresses gravitational instability in the very inner portion of the disk. We found similarly low rates of transport in this region for the no feedback simulations in Paper I. At intermediate radii $1.5 \mathrm{kpc} \lesssim R \lesssim 8 \mathrm{kpc}$, the story is quite different. The mass flux shows alternating patterns of inward and outward transport, which we associate with the formation of spiral arms. We cannot say whether the HGF case shows similar behavior, since it has not run long enough.

The quantity of greatest interest from the standpoint of star formation fueling is not the instantaneous transport rate, but its long term average. We have therefore calculated the timeaveraged mass flux through each radial ring, which we present in Figure 13. The time averaging is conducted in the wedgeshaped regions of radius-time parameter space above the blue lines in Figure 12. This is done to avoid including the time when the disk in undergoing initial collapse and transient behavior. See Paper I for more details.

We see that the rates of inward mass transport, $\sim 0.1 M_{\odot} \mathrm{yr}^{-1}$ in the LGF simulation and $\sim 1 M_{\odot} \mathrm{yr}^{-1}$ in the fiducial one, are comparable to or perhaps slightly smaller what we found in the simulations without feedback (c.f. Figure 7 of Paper I). Thus we conclude that the presence or absence of feedback only modestly affects the rate of mass transport through a galactic disk.

For comparison, we also compute the cumulative timeaveraged star formation interior to each radius, defined simply as the total mass of stars formed whose formation point is interior to that radius in the same wedge-averaging region, divided by the time over which we measure star formation, which is a function of radius. In Figure 13 we show the
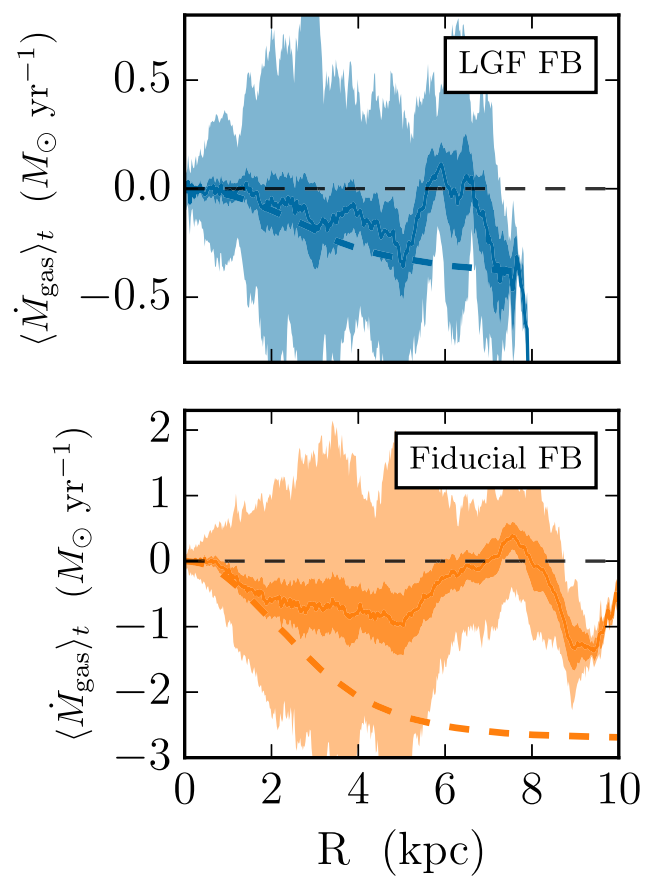

Figure 13. Time-averaged radial mass flux as a function of radius for the low gas fraction (top panel) and fiducial (bottom panel) simulations. At a given radius, the light-shaded region encloses $67 \%$ of the mass flux measurements over the course of the simulation. The dark-shaded region encloses the $1 \sigma$ confidence region for the mean mass flux, which we compute by taking the standard deviation of the mass flux measurements and dividing by the square root of the number of uncorrelated measurements. This measure of the uncertainty in the mean mass flux assumes a correlation timescale of $10 \mathrm{Myr}$, which was measured by eye based on the autocorrelation of the mass flux measurements as a function of time at several radii. The dark, solid line is the mean mass flux. The large variations in mass flux visibly evident in Figure 12 correspond to the significant vertical extent of the light-shaded region. The narrowness of the dark-shaded region indicates that inward net mass transport through the disk is statistically significant. We also plot the negative of the time-averaged radially accumulated star formation rate (dotted-dashed lines) for comparison. At radii where the dashed line and the solid line lie on top of each other, radial mass transport is sufficient to fuel all star formation within that radius.

negative of this quantity in order to ease comparison with the time-averaged mass flux.

At a fixed radius, if the radially cumulative star formation rate is equal to the net inward mass flux, then the mass flux at that radius is sufficient to supply all of the star formation within that radius. For the LGF simulation, we see that over the bulk of the star-forming disk $(R \lesssim 5 \mathrm{kpc})$, radial mass transport is sufficient to supply the bulk of the star formation.

For the fiducial simulation, the mass transport rate is only sufficient to supply the star formation for $R \lesssim 2 \mathrm{kpc}$. Between $2 \mathrm{kpc} \lesssim R \lesssim 5 \mathrm{kpc}$, the radial mass transport rate slowly increases in magnitude from $\sim 0.5 M_{\odot} \mathrm{yr}^{-1}$ up to $\sim 1.0 M_{\odot} \mathrm{yr}^{-1}$ while the radially cumulative star formation rate increases from $\sim 0.5 M_{\odot} \mathrm{yr}^{-1}$ to $\sim 2.5 M_{\odot} \mathrm{yr}^{-1}$. In this region the inward flow of gas is insufficient to sustain star formation, and the gas supply would eventually be substantially depleted if we followed the simulation beyond $600 \mathrm{Myr}$. Further out, the radially cumulative star formation rate only slowly increases to $\sim 2.8 M_{\odot} \mathrm{yr}^{-1}$, while the radial mass transport rate approaches zero, and even becomes slightly positive near $R=8 \mathrm{kpc}$. We caution that the mass transport rates at these radii were measured using a small fraction of the simulation data we have on hand due to the shape of the averaging region (see 
Figure 12). The disk has experienced comparatively little time after initial transient period at these radii so running our simulations for a few more galactic dynamical times may shift the average mass flux.

While the measured mass transport rates in our simulations are not sufficient to supply all of the gas needed for star formation at intermediate radii in the fiducial simulation, it is sufficient to supply a substantial fraction $(\sim 1 / 3)$ of the necessary gas, slowing gas consumption and increasing the depletion time. If we continued the simulation long enough for the gas fraction to decrease to $10 \%$, comparable to the LGF run, then it seems likely that star formation and gas transport would reach full equilibrium as they do in the LGF case. This equilibrium would likely persist until the outer disk was drained of gas.

\section{DISCUSSION AND CONCLUSIONS}

In this paper we have presented three simulations of Milky Way-like disk galaxies under the influence of gravitational instability and star formation feedback. By comparing these results to those we obtained in the absence of stellar feedback (Paper I), we are able to separate out the roles of gravitational instability and stellar feedback in determining the properties of galactic disks, and we are able to study how mass transport might fuel star formation in disk centers.

\subsection{The Effects of Feedback}

We find that feedback is primarily responsible for preventing the ISM from becoming dominated by gravitationally bound clouds, and instead partitioning it into comparable masses of WNM, CNM, and molecular gas with a relatively smooth morphology when averaged over $\sim \mathrm{kpc}$ scales. When we include feedback in our models, we find that our simulated galaxies reach a balance between these phases that is in good agreement with values observed in nearby Milky Way-like disks. Feedback is also responsible for isotropizing the turbulence in galactic disks and thereby pumping up their scale heights compared to what would be expected in its absence. Most importantly, feedback is required to suppress the star formation rate and produce disks with molecular gas depletion times $t_{\mathrm{dep}} \sim 2 \mathrm{Gyr}$, comparable to what is observed in local disks.

On the other hand, feedback is not the main agent determining either the velocity structure or gravitational stability of the ISM. Contrary to naive expectations, including feedback actually reduces the total velocity dispersion and Toomre $Q_{\text {total }}$ parameter of a galactic disk. In the absence of feedback, gravitational instability is fully capable of maintaining large velocity dispersions and preventing $Q_{\text {total }}$ from dipping below unity. Feedback does prevent the formation of local regions where $Q_{\text {total }}<1$-in the simulations with no feedback these regions correspond to the locations of massive star-forming clouds. Feedback disrupts star-forming clouds before enough mass is able to accumulate locally to drive $Q_{\text {total }}$ to values below unity. However, when we compute global averages, the effect of feedback is to lower $Q_{\text {total }}$ and not raise it.

As we saw in Section 3.4.1, the time-averaged azimuthally averaged effective sound speed is suppressed somewhat compared to the simulations with no feedback. For example, compare Figure 8 with Figure 9 in Paper I. This can be explained by examining the time evolution of the effective sound speed in both simulations, as plotted in Figures 11 and 5 of Paper I. In the first $200 \mathrm{Myr}$ of the simulations with no feedback, the azimuthally averaged effective sound speed is initially very similar, albeit somewhat suppressed, compared to the simulations with feedback. Later on, as the gas supply is exhausted, the effective sound speed increases. The increase in the effective sound speed works in concert with the decrease in the gas surface density, increasing $Q_{\text {gas }}$ throughout the starforming portion of the gaseous disk.

In the simulations with feedback, the gas consumption timescale is substantially longer, and the gas surface density profile does not vary much over the course of the simulation. The lower turbulent velocity dispersions in the simulation with feedback are therefore a reflection of the prolonged period of marginal gravitational stability. Eventually, if the simulations were allowed to run for another $10^{9}$ years, and no new gas was added by cosmological accretion, we would expect the gas surface density to decrease, and turbulent velocity dispersion to increase, matching the behavior seen in the simulations with no feedback.

This finding strongly undermines the central assumption made in analytic models that attempt to derive a star formation rate by deducing a value required to maintain $Q_{\text {total }} \approx 1$ (e.g., Faucher-Giguère et al. 2013). It appears that the star formation rate and the maintenance of marginal gravitational stability are physically decoupled phenomena. It is also problematic for models that derive a star formation rate from vertical hydrostatic equilibrium without requiring that $Q_{\text {total }} \approx 1$ (e.g., Ostriker et al. 2010; Ostriker \& Shetty 2011); these models derive the star formation rate from vertical force balance, which is reasonable in light of our finding that feedback is the main agent responsible for setting galaxies' vertical scale heights. On the other hand, these models also posit that galaxies with high surface densities reach $Q_{\text {total }} \ll 1$, which we find that gravitational instability always prevents.

\subsection{Galactic Winds}

Our simulations do not produce substantial galactic winds. This may be surprising given the prominent role winds are thought to play in galaxy evolution (Veilleux et al. 2005; Peeples et al. 2014, and references therein). We see two possible explanations for why our simulations do not produce substantial winds.

First, recent simulations by the FIRE collaboration (Muratov et al. 2015) - cosmological zoom-in simulations with a feedback recipe capable of producing strong winds-indicate that galaxies with masses comparable to the Milky Way do not produce substantial winds at low redshift. Going from high redshift to low redshift, their $\mathrm{m} 12 \mathrm{i}$ halo transitions from a bursty mode of star formation to a mode where stars form at a steady rate. The authors of this study conclude that the fall-off in the rate of mass ejected by winds is due to the deepening of the gravitational potential, less concentrated star formation, and less bursty star formation. All three effects conspire to reduce the effectiveness of gas acceleration out of the halo. In a posthoc analytical investigation of the lack of winds seen at late times in the FIRE simulations, Hayward \& Hopkins (2015) attribute the fall-off in the outflow rate to the decline in the gas fraction. They predict that the ratio of the outflow rate to the star formation rate falls off exponentially at LGFs. This may 
easily account for the lack of appreciable outflows in the LGF and fiducial simulations.

For the HGF simulation, and possibly also for the others, our simulations may not have sufficient resolution to adequately model the details of the wind acceleration process. In the theoretical models of Mac Low \& McCray (1988), the expanding bubble of hot gas that drives the wind must last long enough so that radiative losses cannot drain enough energy for the wind to stall. In particular, the cooling timescale must be longer than the time for the bubble radius to exceed a galactic scale height. In practice, this requires hot gas, with temperatures exceeding $10^{7} \mathrm{~K}$. The $\mathrm{SN}$ bubbles in our simulations do not create gas that is so hot. This is proximately due to the implementation of our feedback recipe, which uses most of the energy of the SN explosions for direct momentum injection rather than the injection of thermal energy. As discussed in Section 2.2.4, we take this approach to avoid the overcooling problem, namely that a single $\mathrm{SN}$ explosion must spread its energy over a volume of $8000 \mathrm{pc}^{3}$ given our resolution, which will typically not heat the gas to a high enough temperature to prevent it from cooling quickly. At the resolution of our simulations, it is therefore difficult to avoid the result that our $\mathrm{SNe}$ produce relatively little hot gas.

This issue is more prominent for us than it is for the FIRE simulations (Muratov et al. 2015) discussed above because, although their resolution is no better than ours, they adopt a star formation recipe that converts all gravitationally bound gas into stars in a single dynamical time. In contrast, we adopt an observationally motivated recipe whereby only $\sim 1 \%$ of the mass per dynamical time is converted to stars (see Paper I for details). This is relevant for galactic winds because it means that the FIRE simulations have much lower densities in the regions where $\mathrm{SNe}$ explode than we find, which in turn greatly reduces the resolution required to properly capture the adiabatic phase of SN remnant evolution.

\subsection{Implications for Star Formation Fueling}

Perhaps the most striking conclusion that can be drawn from our simulations from the standpoint of galaxy evolution concerns star formation fueling. We find that gravitational instability drives gas inflows through galactic disks regardless of whether we include feedback in our simulations or not; feedback only very modestly reduces the inflow rates, even as it completely disperses the large, bound clumps that dominate our disks in the simulations without feedback. This means that inflow is not limited to clumpy high-redshift disks (e.g., Dekel et al. 2009), but is instead a ubiquitous phenomenon that persists to $z=0$. Once we include feedback in our simulations, thereby lowering the star formation rates we measure, this inflow is sufficient to power all of the present-day star formation in a Milky Way-mass galaxy with a $10 \%$ gas fraction, and to power all of the star formation in the core of a galaxy with a $20 \%$ gas fraction and a significant fraction of the star formation at larger radii. This answers the question of why galaxy centers in Milky Way mass galaxies are usually not devoid of gas and star formation. Even though these regions have depletion times much less than a Hubble time, they can be re-supplied from larger radii. This lowers the rate at which the gas fractions drop, and produces a full equilibrium between consumption and infall once the gas fraction is low enough, $\sim 10 \%$. This equilibrium can presumably last as long as there is sufficient gas available at large galactocentric radii, a condition that can be satisfied for $\sim 1$ Hubble time or longer even in the absence of resupply from outside the galaxy.

This work utilized the Hyades supercomputer at the University of California Santa Cruz, which is supported by the National Science Foundation through award AST-1229745, and the Pleiades supercomputer, which is supported by the NASA Advanced Supercomputing Division. The computations and analysis described in this paper rely heavily on open source software packages, including Enzo, Python, yt, IPython, NumPy, SciPy, matplotlib, Cython, hof5, h5py, scikit-image, and numexpr. We thank the developer communities of these packages for their contributions. We also thank the anonymous referee for providing comments that substantially improved the quality of this manuscript. This work was supported by NSF graduate fellowships (NJG and JCF), by NSF grants AST-0955300, AST-1405962 (MRK, NJG, and JCF), and ACI-1535651 (NJG), by NASA TCAN grant NNX14AB52G (MRK, NJG, and JCF), by Hubble Archival Research grant HST-AR-13909 (JCF and MRK), by grant DP16010100695 from the Australian Research Council (MRK), and by the Gordon and Betty Moore Foundation's Data-Driven Discovery Initiative through Grant GBMF4651 to Matthew Turk.

\section{REFERENCES}

Agertz, O., Lake, G., Teyssier, R., et al. 2009, MNRAS, 392, 294 Agertz, O., Romeo, A. B., \& Grisdale, K. 2015, MNRAS, 449, 2156 Bigiel, F., \& Blitz, L. 2012, ApJ, 756, 183

Bigiel, F., Leroy, A., Walter, F., et al. 2008, AJ, 136, 2846

Bigiel, F., Leroy, A. K., Walter, F., et al. 2011, ApJL, 730, L13

Bonnell, I. A., Dobbs, C. L., \& Smith, R. J. 2013, MNRAS, 430, 1790

Bournaud, F., Elmegreen, B. G., Teyssier, R., Block, D. L., \& Puerari, I. 2010, MNRAS, 409, 1088

Bresolin, F., Kennicutt, R. C., \& Ryan-Weber, E. 2012, ApJ, 750, 122

Bresolin, F., Ryan-Weber, E., Kennicutt, R. C., \& Goddard, Q. 2009, ApJ, 695,580

Cacciato, M., Dekel, A., \& Genel, S. 2012, MNRAS, 421, 818

Cen, R., \& Ostriker, J. P. 1992, ApJL, 399, L113

Cioffi, D. F., McKee, C. F., \& Bertschinger, E. 1988, ApJ, 334, 252

Daddi, E., Elbaz, D., Walter, F., et al. 2010, ApJL, 714, L118

Dekel, A., Sari, R., \& Ceverino, D. 2009, ApJ, 703, 785

Dobbs, C. L., Burkert, A., \& Pringle, J. E. 2011, MNRAS, 417, 1318

Dutton, A. A. 2012, MNRAS, 424, 3123

Faucher-Giguère, C.-A., Quataert, E., \& Hopkins, P. F. 2013, MNRAS, 433, 1970

Ferguson, A. M. N., \& Clarke, C. J. 2001, MNRAS, 325, 781

Field, G. B., Goldsmith, D. W., \& Habing, H. J. 1969, ApJL, 155, L149

Forbes, J., Krumholz, M., \& Burkert, A. 2012, ApJ, 754, 48

Forbes, J. C., Krumholz, M. R., Burkert, A., \& Dekel, A. 2014, MNRAS, 438, 1552

Forbes, J. C., Krumholz, M. R., Goldbaum, N. J., \& Dekel, A. 2016, Natur, in press (arXiv:1605.00650)

Fraternali, F., Marasco, A., Marinacci, F., \& Binney, J. 2013, ApJL, 764, L21 Goldbaum, N. J., Krumholz, M. R., \& Forbes, J. C. 2015, ApJ, 814, 131

Hayward, C. C., \& Hopkins, P. F. 2015, arXiv:1510.05650

Ianjamasimanana, R., de Blok, W. J. G., Walter, F., \& Heald, G. H. 2012, AJ, 144,96

Ianjamasimanana, R., de Blok, W. J. G., Walter, F., et al. 2015, AJ, 150, 47

Joung, M. K. R., \& Mac Low, M.-M. 2006, ApJ, 653, 1266

Joung, M. R., Mac Low, M.-M., \& Bryan, G. L. 2009, ApJ, 704, 137

Katz, N. 1992, ApJ, 391, 502

Kennicutt, R. C., \& Evans, N. J. 2012, ARA\&A, 50, 531

Kennicutt, R. C., Jr. 1998, ApJ, 498, 541

Kim, C.-G., Kim, W.-T., \& Ostriker, E. C. 2011a, ApJ, 743, 25

Kim, C.-G., \& Ostriker, E. C. 2015, ApJ, 802, 99

Kim, J.-h., Wise, J. H., Alvarez, M. A., \& Abel, T. 2011b, ApJ, 738, 54

Kimm, T., \& Cen, R. 2014, ApJ, 788, 121

Kimm, T., Cen, R., Devriendt, J., Dubois, Y., \& Slyz, A. 2015, MNRAS, 451, 2900 
Krumholz, M., \& Burkert, A. 2010, ApJ, 724, 895

Krumholz, M. R. 2014, PhR, 539, 49

Krumholz, M. R., \& McKee, C. F. 2005, ApJ, 630, 250

Krumholz, M. R., McKee, C. F., \& Tumlinson, J. 2008, ApJ, 689, 865

Krumholz, M. R., McKee, C. F., \& Tumlinson, J. 2009, ApJ, 693, 216

Leitherer, C., Ekström, S., Meynet, G., et al. 2014, ApJS, 212, 14

Leitherer, C., Schaerer, D., Goldader, J. D., et al. 1999, ApJS, 123, 3

Leroy, A. K., Walter, F., Brinks, E., et al. 2008, AJ, 136, 2782

Leroy, A. K., Walter, F., Sandstrom, K., et al. 2013, AJ, 146, 19

Mac Low, M.-M., \& McCray, R. 1988, ApJ, 324, 776

Martizzi, D., Faucher-Giguère, C.-A., \& Quataert, E. 2015, MNRAS, 450, 504

McKee, C. F., \& Krumholz, M. R. 2010, ApJ, 709, 308

Meidt, S. E., Schinnerer, E., García-Burillo, S., et al. 2013, ApJ, 779, 45

Mo, H. J., Mao, S., \& White, S. D. M. 1998, MNRAS, 295, 319

Muratov, A. L., Kereš, D., Faucher-Giguère, C.-A., et al. 2015, MNRAS, 454, 2691

Olivier, S. S., Primack, J. R., \& Blumenthal, G. R. 1991, MNRAS, 252, 102

Onodera, S., Kuno, N., Tosaki, T., et al. 2010, ApJL, 722, L127

Oppenheimer, B. D., \& Davé, R. 2008, MNRAS, 387, 577

Ostriker, E. C., McKee, C. F., \& Leroy, A. K. 2010, ApJ, 721, 975

Ostriker, E. C., \& Shetty, R. 2011, ApJ, 731, 41

Parravano, A., Hollenbach, D. J., \& McKee, C. F. 2003, ApJ, 584, 797

Peeples, M. S., Werk, J. K., Tumlinson, J., et al. 2014, ApJ, 786, 54

Petit, A. C., Krumholz, M. R., Goldbaum, N. J., \& Forbes, J. C. 2015, MNRAS, 449, 2588
Renaud, F., Bournaud, F., Emsellem, E., et al. 2013, MNRAS, 436, 1836

Romeo, A. B., \& Wiegert, J. 2011, MNRAS, 416, 1191

Scannapieco, C., Tissera, P. B., White, S. D. M., \& Springel, V. 2006, MNRAS, 371, 1125

Schruba, A., Leroy, A. K., Walter, F., Sandstrom, K., \& Rosolowsky, E. 2010, ApJ, 722, 1699

Simpson, C. M., Bryan, G. L., Hummels, C., \& Ostriker, J. P. 2014, arXiv: 1410.3822

Smith, B. D., Hallman, E. J., Shull, J. M., \& O'Shea, B. W. 2011, ApJ, 731, 6

Springel, V., Di Matteo, T., \& Hernquist, L. 2005, MNRAS, 361, 776

Springel, V., \& Hernquist, L. 2003, MNRAS, 339, 289

Stinson, G., Seth, A., Katz, N., et al. 2006, MNRAS, 373, 1074

Tamburro, D., Rix, H.-W., Leroy, A. K., et al. 2009, AJ, 137, 4424

Tasker, E. J., \& Bryan, G. L. 2008, ApJ, 673, 810

The Enzo Collaboration, Bryan, G. L., Norman, M. L., O'Shea, B. W., et al. 2014, ApJS, 211, 19

Thompson, T. A., Quataert, E., \& Murray, N. 2005, ApJ, 630, 167

Toomre, A. 1964, ApJ, 139, 1217

Vázquez, G. A., \& Leitherer, C. 2005, ApJ, 621, 695

Veilleux, S., Cecil, G., \& Bland-Hawthorn, J. 2005, ARA\&A, 43, 769

Werk, J. K., Putman, M. E., Meurer, G. R., \& Santiago-Figueroa, N. 2011, ApJ, 735, 71

Wolfire, M. G., McKee, C. F., Hollenbach, D., \& Tielens, A. G. G. M. 2003, ApJ, 587, 278

Yang, C.-C., \& Krumholz, M. 2012, ApJ, 758, 48 